\title{
Local Stereology of Tensors of Convex Bodies
}

\author{
Eva B. Vedel Jensen • Johanna F. Ziegel
}

Received: 26 September 2012 / Revised: 1 February 2013 /

Accepted: 1 April 2013 / Published online: 17 April 2013

(C) Springer Science+Business Media New York 2013

\begin{abstract}
In this paper, we present local stereological estimators of Minkowski tensors defined on convex bodies in $\mathbb{R}^{d}$. Special cases cover a number of well-known local stereological estimators of volume and surface area in $\mathbb{R}^{3}$, but the general setup also provides new local stereological estimators of various types of centres of gravity and tensors of rank two. Rank two tensors can be represented as ellipsoids and contain information about shape and orientation. The performance of some of the estimators of centres of gravity and volume tensors of rank two is investigated by simulation.
\end{abstract}

Keywords Ellipsoidal approximation - Local stereology • Minkowski tensors • Particle shape $\cdot$ Particle orientation $\cdot$ Rotational integral geometry

AMS 2000 Subject Classification $60 \mathrm{D} 05 \cdot 53 \mathrm{C} 65 \cdot 52 \mathrm{~A} 22$

\section{Introduction}

Local stereology provides estimators of mean particle size without restrictive assumptions about particle shape. It is also possible to stereologically estimate the particle size distribution without shape assumptions when the size of the individually sampled particles is determined with sufficient precision (Pawlas et al. 2009).

\footnotetext{
E. B. Vedel Jensen

Department of Mathematics, Aarhus University, Ny Munkegade 118, 8000 Aarhus C, Denmark

e-mail: eva@imf.au.dk

J. F. Ziegel (凶)

Department of Mathematics and Statistics, Institute of Mathematical Statistics and Acturarial Science, University of Bern, Sidlerstrasse 5, 3012 Bern, Switzerland e-mail: johanna.ziegel@stat.unibe.ch
} 
However, it is still an open question how to estimate stereologically particle shape and orientation without initially restricting to a specific class of shapes. A global stereological procedure for estimating anisotropy is available (Cruz-Orive et al. 1985), but otherwise the focus in the stereological literature has mainly been on estimating shape and orientation of ellipsoidal particles. For the cases of either prolate or oblate ellipsoids, stereological methods of estimating the bivariate distribution of the lengths of the two semiaxes from sectional data were already developed by Cruz-Orive (1976, 1978). Further developments in this direction include Gokhale (1996); Beneš et al. (1997). But, to the best of our knowledge, the general case of stereologically estimating shape of arbitrary particles has remained an open problem.

In the present paper, we take up this problem. We associate to each particle a system of Minkowski tensors and develop local stereological methods of estimating these tensors. The rank zero Minkowski tensors are simply the intrinsic volumes for which local stereological estimation procedures have already been developed. The rank one tensors provide information about centres of gravity of different types. Rank two tensors can naturally be associated to ellipsoids and contain information about the shape and orientation of the original particle.

Minkowski tensors have been used with success in material science (Beisbart et al. 2002; Denis et al. 2008; Schröder-Turk et al. 2011a, b), but there are also examples from the biosciences (Beisbart et al. 2006). In these applications, the structure is observed directly. In the present paper, we use measurements on sections through the particles instead.

The paper is organised as follows. In Section 2, we give a short introduction to Minkowski tensors while Section 3 gives the geometric interpretation of the Minkowski tensors as descriptors of size, shape and orientation. The local stereological estimators of Minkowski tensors are derived in Section 4 and all the special cases in 3D are discussed in Section 5. A simulation study is presented in Section 6. Perspectives and future research are discussed in Section 7.

\section{Minkowski Tensors}

This section contains a condensed introduction to Minkowski tensors. For a more comprehensive treatment, see Hug et al. (2008) and references therein.

Let $X \subseteq \mathbb{R}^{d}$ be a convex and compact subset of $\mathbb{R}^{d}$. For a non-negative integer $r$, the volume tensor of rank $r$ is defined by

$$
\Phi_{d, r, 0}(X)=\frac{1}{r !} \int_{X} x^{r} \lambda_{d}(\mathrm{~d} x)
$$

where $x^{r}$ is the tensor of rank $r$ determined by $x$ and $\lambda_{d}$ is the Lebesgue measure on $\mathbb{R}^{d}$.

For $k=0, \ldots, d-1$ and non-negative integers $r$ and $s$, let

$$
\Phi_{k, r, s}(X)=\frac{\omega_{d-k}}{r ! s ! \omega_{d-k+s}} \int_{\mathbb{R}^{d} \times \mathbb{S}^{d-1}} x^{r} u^{s} \Lambda_{k}(X, \mathrm{~d}(x, u)),
$$

where $\omega_{d}=2 \pi^{d / 2} / \Gamma(d / 2)$ is the surface area of $\mathbb{S}^{d-1}$ and $\Lambda_{k}(X, \cdot)$ is the $k$ th support measure or generalised curvature measure of $X, k=0, \ldots, d-1$. Furthermore, $x^{r} u^{s}$ is the symmetric tensor product of rank $r+s$ of $x^{r}$ and $u^{s}$. 
The support measures $\Lambda_{k}(X, \cdot), k=0, \ldots, d-1$, are concentrated on the normal bundle Nor $X$ of $X$ which consists of all pairs $(x, u)$ where $x \in \partial X$ and $u$ is an outer unit normal vector of $X$ at $x$. For this reason, the tensors defined in Eq. 2 are called surface tensors. For $r=s=0, \Phi_{k, 0,0}=V_{k}$, the $k$ th intrinsic volume, $k=0, \ldots, d$.

If $\partial X$ is a regular hypersurface of class $C^{2}$, then Eq. 2 reduces to

$$
\Phi_{k, r, s}(X)=\frac{\omega_{d-k}}{r ! s ! \omega_{d-k+s}} \int_{\partial X} x^{r} u(x)^{s} \Phi_{k}(X, \mathrm{~d} x),
$$

where $u(x)$ is the unique outer unit normal vector of $X$ at $x$ and $\Phi_{k}(X, \cdot)$ is the $k$ th curvature measure of $X, k=0, \ldots, d-1$. For $k=d-1$,

$$
2 \Phi_{d-1}(X, A)=\mathcal{H}^{d-1}(A \cap \partial X),
$$

where $\mathcal{H}^{d-1}$ is the $(d-1)$-dimensional Hausdorff measure, and Eq. 3 takes the simple form

$$
\Phi_{d-1, r, s}(X)=\frac{\omega_{d-k}}{2 r ! s ! \omega_{d-k+s}} \int_{\partial X} x^{r} u(x)^{s} \mathcal{H}^{d-1}(\mathrm{~d} x) .
$$

The tensors defined in Eqs. 1 and 2 constitute the Minkowski tensors. They are tensor-valued valuations, continuous with respect to the Hausdorff metric and motion covariant.

\section{Minkowski Tensors as Descriptors of Size, Shape and Orientation of Convex Bodies}

Minkowski tensors of rank zero are simply the intrinsic volumes and as such contain primarily size information. Minkowski tensors of rank one give information about different types of centres of gravity of the convex body in question, i.e. information about position. Minkowski tensors of rank two provide additional information about shape and orientation of the convex body.

To make the latter statement more explicit, let us focus on volume tensors of rank two. They are defined by the following equation

$$
\Phi_{d, 2,0}(X)=\frac{1}{2} \int_{X} x^{2} \lambda_{d}(\mathrm{~d} x) .
$$

Now, suppose that $X$ is an ellipsoid centred at the origin. Then, there exists a symmetric positive definite matrix $A$ such that

$$
X=\left\{A w: w \in \mathbb{B}^{d}\right\},
$$

where $\mathbb{B}^{d}$ is the unit ball in $\mathbb{R}^{d}$. In this case, we have

$$
\Phi_{d, 2,0}(X)=\frac{|A| \kappa_{d}}{2(d+2)} A A^{*},
$$

where $\kappa_{d}=\pi^{d / 2} / \Gamma(1+d / 2)$ is the volume of $\mathbb{B}^{d},|A|$ denotes the determinant of $A$ and $A^{*}$ its transpose. If we let $A=B \Lambda B^{*}$ be the (unique) spectral decomposition of $A$ where $B$ is an orthogonal matrix and $\Lambda$ a diagonal matrix, we can regard $X$ as being obtained by rotating an ellipsoid with axes parallel to the coordinate axes with the rotation $B$. The ellipsoid has semiaxis lengths equal to the diagonal elements $\lambda_{i}$, 
$i=1, \ldots, d$, of $\Lambda$. Using this decomposition, we can rewrite the volume tensor as follows

$$
\Phi_{d, 2,0}(X)=\frac{\kappa_{d} \prod_{i=1}^{d} \lambda_{i}}{2(d+2)} B \Lambda^{2} B^{*} .
$$

Clearly, we can (uniquely) reconstruct $X$ from its volume tensor. The volume tensor will have a spectral decomposition of the form

$$
\Phi_{d, 2,0}(X)=B \tilde{\Lambda} B^{*}
$$

with the same rotation matrix as $A$ and a diagonal matrix with elements $\tilde{\lambda}_{i}$ satisfying

$$
\tilde{\lambda}_{i}=\frac{\kappa_{d} \prod_{j=1}^{d} \lambda_{j}}{2(d+2)} \lambda_{i}^{2}, \quad i=1, \ldots, d .
$$

Solving these equations we obtain

$$
\lambda_{i}=\left(\frac{2(d+2)}{\kappa_{d}}\right)^{\frac{1}{d+2}} \frac{\tilde{\lambda}_{i}^{\frac{d+1}{2(d+2)}}}{\prod_{j \neq i} \tilde{\lambda}_{j}^{\frac{1}{2(d+2)}}} .
$$

If $X$ is not an ellipsoid centred at the origin, we may still determine its volume tensor of rank two and associate to $X$ an approximating ellipsoid. Below, we give two examples.

Example 1 Let $a_{i}>0, i=1, \ldots, d$, and let $X=\left[-a_{1}, a_{1}\right] \times \cdots \times\left[-a_{d}, a_{d}\right]$ be a centred box. Then, $\Phi_{d, 2,0}(X)$ is a diagonal matrix with

$$
\Phi_{d, 2,0}(X)_{i i}=\tilde{\lambda}_{i}=\frac{2^{d-1}}{3} a_{i}^{3} \prod_{j \neq i} a_{j}, \quad i=1, \ldots, d .
$$

It follows that $X$ is approximated by an ellipsoid with axes parallel to the coordinate axes. The length of the $i$ th semiaxis of this approximating ellipsoid is

$$
\lambda_{i}=2^{\frac{d}{d+2}}\left(\frac{d+2}{3 \kappa_{d}}\right)^{\frac{1}{d+2}} a_{i}, \quad i=1, \ldots, d .
$$

Example 2 Let $r, h>0$ and let $X$ be a centred spherical cylinder

$$
X=\left\{x \in \mathbb{R}^{d}: \sqrt{x_{1}^{2}+\cdots+x_{d-1}^{2}} \leq r,\left|x_{d}\right| \leq h\right\} .
$$

Then, $\Phi_{d, 2,0}(X)$ is a diagonal matrix with

$$
\Phi_{d, 2,0}(X)_{i i}= \begin{cases}\frac{\pi^{\frac{d-1}{2}}}{2 \Gamma\left(\frac{d+3}{2}\right)} h r^{d+1}, & i<d, \\ \frac{\pi^{\frac{d-1}{2}}}{3 \Gamma\left(\frac{d+1}{2}\right)} h^{3} r^{d-1}, & i=d .\end{cases}
$$


It follows that $X$ is approximated by an ellipsoid with axes parallel to the coordinate axes. The lengths of the semiaxes of this approximating ellipsoid are

$$
\lambda_{i}= \begin{cases}\left(\frac{\sqrt{3}(d+2) \Gamma\left(\frac{d+2}{2}\right)}{\sqrt{\pi} \sqrt{d+1} \Gamma\left(\frac{d+3}{2}\right)}\right)^{\frac{1}{d+2}} r, & i<d, \\ \left(\frac{d+1}{3}\right)^{\frac{d+1}{2(d+2)}}\left(\frac{(d+2) \Gamma\left(\frac{d+2}{2}\right)}{\sqrt{\pi} \Gamma\left(\frac{d+3}{2}\right)}\right)^{\frac{1}{d+2}} h, & i=d .\end{cases}
$$

\section{Local Stereological Estimators of Minkowski Tensors}

Auneau-Cognacq et al. (2013) have derived a new rotational Crofton formula for so-called integrated Minkowski tensors. Special cases of this formula express the classical Minkowski tensors as rotational averages; see Auneau-Cognacq et al. (2013, Corollary 4.4).

In this section, we will use this result to derive local stereological estimators of Minkowski tensors based on observation in an isotropic $p$-subspace $L_{p}$, i.e. a random $p$-dimensional linear subspace in $\mathbb{R}^{d}$ with distribution determined by a normalised version of the rotation invariant measure on the set $\mathcal{L}_{p}^{d}$ of all $p$-subspaces in $\mathbb{R}^{d}$. The rotation invariant measure on $\mathcal{L}_{p}^{d}$ will be denoted by $\mathrm{d} L_{p}^{d}$. Its total mass is chosen to be

$$
\int_{\mathcal{L}_{p}^{d}} \mathrm{~d} L_{p}^{d}=c_{d, p},
$$

where

$$
c_{d, p}=\frac{\omega_{d} \omega_{d-1} \cdots \omega_{d-p+1}}{\omega_{p} \omega_{p-1} \cdots \omega_{1}} .
$$

The isotropic probability measure on $p$-subspaces is thus $\mathrm{d} L_{p}^{d} / c_{d, p}$.

The local stereological estimators of Minkowski tensors presented in the proposition below are formulated in terms of integrals on the section $X \cap L_{p}$. The integrals are with respect to affine subspaces of dimension $p-1$ within $L_{p}$. The set of such affine subspaces is denoted by $\mathcal{F}_{p-1}^{p}$. The measure $\mathrm{d} F_{p-1}^{p}$ used in the integration is the motion invariant measure on $\mathcal{F}_{p-1}^{p}$. If $F_{p-1}=x+L_{p-1}$, where $L_{p-1}$ is a $(p-1)$ subspace in $L_{p}$ and $x \in L_{p-1}^{\perp} \cap L_{p}$, then $\mathrm{d} F_{p-1}^{p}=\lambda_{1}(\mathrm{~d} x) \mathrm{d} L_{p-1}^{p}$.

At first sight, the formulation in Proposition 1 below may appear to be a very indirect way of expressing the local stereological estimators of Minkowski tensors. It turns out, however, that this approach is very powerful because it is general and it also points to how to determine the estimators in the sections. However, in many special cases much more explicit formulae are available, as we shall see in the next section. 
Proposition 1 Let $X$ be a convex body in $\mathbb{R}^{d}$ and let $L_{p}$ be an isotropic p-subspace. Then, for $0<m<p \leq d$, a non-negative integer $r$ and $s \in\{0,1\}$

$$
\begin{aligned}
& \widehat{\Phi}_{d+m-p, r, s}\left(X ; L_{p}\right) \\
& =\frac{c_{d, p}}{a_{d, p-1, m-1, s}} \int_{\mathcal{F}_{p-1}^{p}} \Phi_{m-1, r, s}^{\left(F_{p-1}\right)}\left(X \cap F_{p-1}\right) d\left(F_{p-1}, O\right)^{d-p} \mathrm{~d} F_{p-1}^{p}
\end{aligned}
$$

is an unbiased estimator of $\Phi_{d+m-p, r, s}(X)$. Here,

$$
a_{d, p-1, m-1, s}=c_{d-1, p-2} \frac{(p-2) !(d+m-p) !}{(m-1) !(d-1) !} \frac{\omega_{d+m-p+s+2}}{\omega_{m+s+1}},
$$

the upper index of $\Phi_{m-1, r, s}^{\left(F_{p-1}\right)}$ indicates that the Minkowski tensor is determined relative to $F_{p-1}$ and $d\left(F_{p-1}, O\right)$ is the distance of the affine subspace $F_{p-1}$ to the origin $O$.

Furthermore, for $0<p \leq d$ and any non-negative integer $r$

$$
\widehat{\Phi}_{d, r, 0}\left(X ; L_{p}\right)=\frac{c_{d, p}}{c_{d, p-1}} \int_{\mathcal{F}_{p-1}^{p}} \Phi_{p-1, r, 0}^{\left(F_{p-1}\right)}\left(X \cap F_{p-1}\right) d\left(F_{p-1}, O\right)^{d-p} \mathrm{~d} F_{p-1}^{p}
$$

is an unbiased estimator of $\Phi_{d, r, 0}(X)$.

Proof The result follows from the rotational integral geometric identities presented in Auneau-Cognacq et al. (2013, Corollary 4.4). Thus, if we let $q=1$ in AuneauCognacq et al. (2013, (14)), we find for $0<m<p \leq d$, a non-negative integer $r$ and $s \in\{0,1\}$,

$$
\begin{aligned}
& \Phi_{d+m-p, r, s}(X) \\
& \quad=\frac{1}{a_{d, p-1, m-1, s}} \int_{\mathcal{L}_{p}^{d}} \int_{\mathcal{F}_{p-1}^{p}} \Phi_{m-1, r, s}^{\left(F_{p-1}\right)}\left(X \cap F_{p-1}\right) d\left(F_{p-1}, O\right)^{d-p} \mathrm{~d} F_{p-1}^{p} \mathrm{~d} L_{p}^{d} .
\end{aligned}
$$

It follows that $\widehat{\Phi}_{d+m-p, r, s}(X)$ in Eq. 4 is an unbiased estimator of $\Phi_{d+m-p, r, s}(X)$. Similarly, if we let $q=1$ in Auneau-Cognacq et al. (2013, (15)), it is seen that $\widehat{\Phi}_{d, r, 0}\left(X ; L_{p}\right)$ in Eq. 5 is an unbiased estimator of $\Phi_{d, r, 0}\left(X ; L_{p}\right)$ for $1<p \leq d$. In the case $p=1$, Eq. 5 reduces to

$$
\widehat{\Phi}_{d, r, 0}\left(X ; L_{1}\right)=c_{d, 1} \frac{1}{r !} \int_{X \cap L_{1}} x^{r} d(x, O)^{d-1} \lambda_{1}(\mathrm{~d} x) .
$$

The unbiasedness of this estimator is a consequence of polar decomposition of Lebesgue measure in $\mathbb{R}^{d}$

$$
\lambda_{d}(\mathrm{~d} x)=d(x, O)^{d-1} \lambda_{1}(\mathrm{~d} x) \mathrm{d} L_{1}^{d} .
$$

A simplified expression for the estimator $\widehat{\Phi}_{d, r, 0}\left(X ; L_{p}\right)$ can be obtained by using Auneau-Cognacq et al. (2013, Proposition 5.1).

Corollary 1 The estimator in Eq. 5 has the following more explicit form

$$
\widehat{\Phi}_{d, r, 0}\left(X ; L_{p}\right)=\frac{c_{d, p}}{c_{d-1, p-1}} \frac{1}{r !} \int_{X \cap L_{p}} x^{r} d(x, O)^{d-p} \lambda_{p}(\mathrm{~d} x) .
$$


Proof Using Auneau-Cognacq et al. (2013, Proposition 5.1) with $(d, j, t)=(p, p-$ $1, d-p$ ), we find

$$
\begin{aligned}
\widehat{\Phi}_{d, r, 0}\left(X ; L_{p}\right) & =\frac{c_{d, p}}{c_{d, p-1}} \frac{c_{p, p-1}}{r !} \frac{\Gamma\left(\frac{d-p+1}{2}\right) \Gamma\left(\frac{p}{2}\right)}{\Gamma\left(\frac{d}{2}\right) \Gamma\left(\frac{1}{2}\right)} \int_{X \cap L_{p}} x^{r} d(x, O)^{d-p} \lambda_{p}(\mathrm{~d} x) \\
& =\frac{c_{d, p}}{c_{d-1, p-1}} \frac{1}{r !} \int_{X \cap L_{p}} x^{r} d(x, O)^{d-p} \lambda_{p}(\mathrm{~d} x) .
\end{aligned}
$$

Likewise, the estimator $\widehat{\Phi}_{d+m-p, r, s}\left(X ; L_{p}\right)$ may also be simplified. For instance, for $s=0$ and $m=p-1, \widehat{\Phi}_{d+m-p, r, s}\left(X ; L_{p}\right)$ can be expressed as an integral over the normal bundle of $X \cap L_{p}$ considered as a subset of $L_{p}$ with respect to the $(p-1)$ th support measure of $X \cap L_{p}$; cf. Auneau-Cognacq et al. (2013, Proposition 5.5). Determining of the estimator in practice, the expression given in Proposition 1 may, however, be more useful because it avoids measurements of angles.

For applications, it is also interesting to derive estimators of Minkowski tensors, based on an isotropic $p$-subspace $L_{p(q)}$ containing a fixed $q$-subspace, $0 \leq q<p$. The set of such subspaces is denoted by $\mathcal{L}_{p(q)}^{d}$. If we let $\mathrm{d} L_{p(q)}^{d}$ be the rotation invariant measure on $\mathcal{L}_{p(q)}^{d}$ with total mass $c_{d-q, p-q}$, the probability distribution of an isotropic $p$-subspace containing a fixed $q$-subspace is given by $\mathrm{d}_{p(q)}^{d} / c_{d-q, p-q}$. Using that

$$
c_{d-1-q, p-1-q} \lambda_{d}(\mathrm{~d} x)=d\left(x, L_{q}\right)^{d-p} \lambda_{p}(\mathrm{~d} x) \mathrm{d} L_{p(q)}^{d},
$$

see e.g. Jensen (1998, Proposition 4.5), it is easy to derive an unbiased estimator of a volume tensor based on such a subspace. The estimator takes the form

$$
\widehat{\Phi}_{d, r, 0}\left(X ; L_{p(q)}\right)=\frac{c_{d-q, p-q}}{c_{d-1-q, p-1-q}} \frac{1}{r !} \int_{X \cap L_{p(q)}} x^{r} d\left(x, L_{q}\right)^{d-p} \lambda_{p}(\mathrm{~d} x) .
$$

\section{Local Estimators in 3D}

In this section, we will show that Proposition 1 covers, as special cases, a number of the well-known local stereological estimators of volume and surface area, but also provides interesting new local stereological estimators of various types of centres of gravity and tensors of rank two.

Throughout this section we assume that $O \in X$. Below, we explore all the estimators provided by Proposition 1 for $d=3$ and $r+s \leq 2$. To the best of our knowledge, the local estimators presented in Sections 5.2 and 5.3 are new.

\subsection{The Case $r+s=0$}

When $d=3$ and $r=s=0$, Proposition 1 provides local estimators of volume from either lines or planes passing through the origin $O(p=1$ or 2 in Eq. 5, respectively) and surface area from planes through $O(p=2$ in Eq. 4). In addition, Eq. 7 provides a local estimator of volume from vertical sections. 


\subsubsection{Volume from Isotropic Random Lines: The Nucleator}

Letting $d=3, r=0, p=1$ in Eq. 5 or, equivalently, in Eq. 6, we find

$$
\widehat{\Phi}_{3,0,0}\left(X ; L_{1}\right)=2 \pi \int_{X \cap L_{1}} d(x, O)^{2} \lambda_{1}(\mathrm{~d} x) .
$$

Since $O \in X$ and $X$ is convex, $X \cap L_{1}=\left[x_{-}, x_{+}\right]$is a line segment containing the origin. It follows that

$$
\widehat{\Phi}_{3,0,0}\left(X ; L_{1}\right)=\frac{2 \pi}{3}\left(d\left(x_{+}, O\right)^{3}+d\left(x_{-}, O\right)^{3}\right) .
$$

If $X$ is a ball centred at the origin $O$, then $\widehat{\Phi}_{3,0,0}\left(X ; L_{1}\right)$ is identically equal to the parameter to be estimated, the volume of the ball.

In practice, measurements along two perpendicular lines in a planar section are performed. This results in an important variance reduction due to an antithetic effect. The estimator was originally suggested by Gundersen (1988) and has been widely used in the biomedical sciences ever since. In the applied literature, the estimator is called the nucleator.

\subsubsection{Volume from Isotropic Random Planes: The Isotropic Rotator}

Let $d=3, r=0, p=2$ in Eq. 5 or, equivalently, in Eq. 6. Then,

$$
\widehat{\Phi}_{3,0,0}\left(X ; L_{2}\right)=2 \int_{X \cap L_{2}} d(x, O) \lambda_{2}(\mathrm{~d} x) .
$$

As for the nucleator, $\widehat{\Phi}_{3,0,0}\left(X ; L_{2}\right)$ is exact for a ball centred at the origin.

This estimator is the method of choice for local volume estimation from isotropic probes if automatic segmentation of particle sections is available because the estimator has smaller variance than the nucleator. A discretised version of the estimator was originally suggested by Jensen and Gundersen (1993) under name of the isotropic rotator. Recently, Cruz-Orive (2012) has shown that $\widehat{\Phi}_{3,0,0}\left(X ; L_{2}\right)$ is identical to the so-called wedge estimator, based on the invariator principle. The estimator can also be obtained from the nucleator by integrating over all rotating lines through $O$ in $L_{2}$. For this reason, the estimator has also been called the integrated nucleator (Hansen et al. 2011).

\subsubsection{Volume from Vertical Random Planes: The Vertical Rotator}

Let $d=3, r=0, p=2, q=1$ in Eq. 7. Then,

$$
\widehat{\Phi}_{3,0,0}\left(X ; L_{2(1)}\right)=\pi \int_{X \cap L_{2(1)}} d\left(x, L_{1}\right) \lambda_{2}(\mathrm{~d} x) .
$$

This estimator is exact if $X$ is a body of revolution around $L_{1}$.

The estimator $\widehat{\Phi}_{3,0,0}\left(X ; L_{2(1)}\right)$ may be discretised, using the following decomposition of Lebesgue measure in $L_{2(1)}$

$$
\widehat{\Phi}_{3,0,0}\left(X ; L_{2(1)}\right)=\pi \int_{X \cap L_{1}} \int_{X \cap L_{2(1)} \cap\left(y+L_{1}^{\perp}\right)} d\left(x, L_{1}\right) \lambda_{1}(\mathrm{~d} x) \lambda_{1}(\mathrm{~d} y) .
$$

The resulting discretised estimator is called the vertical rotator (Jensen and Gundersen 1993). Vertical sections are useful when it is important to keep track of the structure. 


\subsubsection{Surface Area from Isotropic Random Planes: The Flower Estimator}

If we let $d=3, r=s=0, p=2, m=1$ in Eq. 4, we find

$$
\widehat{\Phi}_{2,0,0}\left(X ; L_{2}\right)=2 \int_{\mathcal{F}_{1}^{2}} \mathbb{1}\left\{X \cap F_{1} \neq \emptyset\right\} d\left(F_{1}, O\right) \mathrm{d} F_{1}^{2},
$$

where $\mathbb{1}\{\cdot\}$ denotes the indicator function and the integration is with respect to all lines in $L_{2}$, intersecting $X$. The estimator $\widehat{\Phi}_{2,0,0}\left(X ; L_{2}\right)$ is exact if $X$ is a ball centred at the origin.

Cruz-Orive (2005) has shown that $\widehat{\Phi}_{2,0,0}\left(X ; L_{2}\right)$ is equal to two times the area of the flower set associated with $X \cap L_{2}$. Recall that the flower set has the graph of the support function of $X \cap L_{2}$ as its boundary. So $\widehat{\Phi}_{2,0,0}\left(X ; L_{2}\right)$ may be called the flower estimator.

There are a number of equivalent expressions for this surface area estimator. It follows from Auneau-Cognacq et al. (2013, Proposition 5.5) that in the special case where the boundary of $X$ is smooth

$$
\widehat{\Phi}_{2,0,0}\left(X ; L_{2}\right)=\int_{\partial X \cap L_{2}}[\cos \beta(x)+\beta(x) \sin \beta(x)] d(x, O) \mathcal{H}_{1}(\mathrm{~d} x),
$$

where $\mathcal{H}_{1}$ is the one-dimensional Hausdorff measure (length measure) and $\beta(x)$ is the angle between $\operatorname{span}\{x\}$ and $\operatorname{span}\{u(x)\}$. Recall, that $u(x)$ is the unique outer unit normal vector of $X$ at $x$. This expression for the estimator reveals its intimate relation to another local estimator of surface area, the surfactor, that was suggested 25 years ago by Jensen and Gundersen (1987). The flower estimator can thereby be interpreted as an integrated surfactor (Dvořák and Jensen 2012).

Cruz-Orive (2005) has shown that the flower estimator can also be expressed as

$$
\widehat{\Phi}_{2,0,0}\left(X ; L_{2}\right)=\int_{0}^{2 \pi} h_{X \cap L_{2}}(\theta)^{2} \mathrm{~d} \theta
$$

where $h_{X \cap L_{2}}(\cdot)$ is the support function of $X \cap L_{2}$. A discretisation of the flower estimator based on determination of the support function in both directions along two perpendicular lines in the section plane is very efficient (Dvořák and Jensen 2012). This discretisation is called the pivotal estimator (Cruz-Orive 2008, 2011).

\subsection{The Case $r+s=1$}

When $d=3$ and $r+s=1$, Proposition 1 provides local estimators of $\Phi_{3,1,0}(X)$ from either lines or planes passing through the origin $O(r=1, p=1$ or 2 in Eq. 5 , respectively) and a local estimator of $\Phi_{2,1,0}(X)$ from planes through $O(r=1, s=0$, $m=1, p=2$ in Eq. 4). In addition, Eq. 7 provides a local estimator of $\Phi_{3,1,0}(X)$ from vertical sections.

By combining these estimators with estimators of volume or surface area, we obtain estimators of the usual centre of gravity $\Phi_{3,1,0}(X) / \Phi_{3,0,0}(X)$ and a boundary centre of gravity $\Phi_{2,1,0}(X) / \Phi_{2,0,0}(X)$. Note that, $\Phi_{2,0,1} \equiv O$. 


\subsubsection{Usual Centre of Gravity}

We can estimate $\Phi_{3,1,0}(X)$ from isotropic random lines, isotropic random planes, or vertical random planes, i.e.

$$
\begin{aligned}
\widehat{\Phi}_{3,1,0}\left(X ; L_{1}\right) & =2 \pi \int_{X \cap L_{1}} x d(x, O)^{2} \lambda_{1}(\mathrm{~d} x) \\
\widehat{\Phi}_{3,1,0}\left(X ; L_{2}\right) & =2 \int_{X \cap L_{2}} x d(x, O) \lambda_{2}(\mathrm{~d} x) \\
\widehat{\Phi}_{3,1,0}\left(X ; L_{2(1)}\right) & =\pi \int_{X \cap L_{2(1)}} x d\left(x, L_{1}\right) \lambda_{2}(\mathrm{~d} x)
\end{aligned}
$$

Note that $\widehat{\Phi}_{3,1,0}\left(X ; L_{1}\right)=\widehat{\Phi}_{3,1,0}\left(X ; L_{2}\right)=O$ if $X$ is centrally symmetric about $O$. The estimator $\widehat{\Phi}_{3,1,0}\left(X ; L_{2(1)}\right)$ is exact for a body of revolution around $L_{1}$. Note also that in all cases $\widehat{\Phi}_{3,1,0}\left(X ; L_{p(q)}\right) \in L_{p(q)}, 0 \leq q<p \leq 2$.

The estimator $\widehat{\Phi}_{3,1,0}\left(X ; L_{1}\right)$ can be simplified considerably. Thus, let $e$ be a unit vector spanning $L_{1}$. Then, if the endpoints of the line segment $X \cap L_{1}$ are chosen such that $x_{+}$points in the direction of $e$ while $x_{-}$points in the opposite direction, then

$$
\widehat{\Phi}_{3,1,0}\left(X ; L_{1}\right)=\frac{\pi}{2}\left(d\left(x_{+}, O\right)^{4}-d\left(x_{-}, O\right)^{4}\right) e .
$$

\subsubsection{Boundary Centre of Gravity}

Proposition 1 provides the following local estimator of $\Phi_{2,1,0}(X)$ based on measurements in an isotropic random plane

$$
\widehat{\Phi}_{2,1,0}\left(X ; L_{2}\right)=2 \int_{\mathcal{F}_{1}^{2}}\left(\sum_{x \in \partial\left(X \cap F_{1}\right)} x\right) d\left(F_{1}, O\right) \mathrm{d} F_{1}^{2} .
$$

The integration is with respect to all lines in $L_{2}$, intersecting $X$. Since $X$ is convex, the sum in the integrand involves at most two points. Note that this estimator also has the property $\widehat{\Phi}_{2,1,0}\left(X ; L_{2}\right) \in L_{2}$.

Proposition 1 also provides a local estimator of $\Phi_{2,0,1}(X) \equiv O$. The estimator

$$
\widehat{\Phi}_{2,0,1}\left(X ; L_{2}\right)=2 \int_{\mathcal{F}_{1}^{2}}\left(\sum_{x \in \partial\left(X \cap F_{1}\right)} u(x)\right) d\left(F_{1}, O\right) \mathrm{d} F_{1}^{2}
$$

is also identically equal to $O$ since when $F_{1}$ hits $X$

$$
\left\{u(x): x \in \partial\left(X \cap F_{1}\right)\right\}=\left\{u_{0},-u_{0}\right\},
$$

where $u_{0}$ is a unit vector, spanning $F_{1}$.

\subsection{The Case $r+s=2$}

When $d=3$ and $r+s=2$, Proposition 1 provides local estimators of $\Phi_{3,2,0}(X)$ from either lines or planes passing through the origin $O(r=2, p=1$ or 2 in Eq. 5, respectively) and local estimators of $\Phi_{2,2,0}(X)$ and $\Phi_{2,1,1}(X)$ from planes through $O((r, s)=(2,0)$ or $(1,1)$, respectively, $m=1, p=2$ in Eq. 4). In addition, Eq. 7 provides a local estimator of $\Phi_{3,2,0}(X)$ from vertical sections. 


\subsubsection{Volume Tensors of Rank Two}

The local estimator of the volume tensor of rank two, based on measurements in an isotropic random line through $O$, is given by

$$
\widehat{\Phi}_{3,2,0}\left(X ; L_{1}\right)=\pi \int_{X \cap L_{1}} x^{2} d(x, O)^{2} \lambda_{1}(\mathrm{~d} x) .
$$

With the notation introduced in Section 5.2.1, this estimator reduces to

$$
\widehat{\Phi}_{3,2,0}\left(X ; L_{1}\right)=\frac{\pi}{5}\left(d\left(x_{+}, O\right)^{5}+d\left(x_{-}, O\right)^{5}\right) e^{2},
$$

which is a singular random matrix.

It is no surprise that two measurements on a single random line are not enough to construct a reliable estimate of the volume tensor of rank two which is a $3 \times 3$ symmetric matrix that contains 6 parameters. One would expect that two measurements from at least three lines are needed. Thus, let us consider three isotropic, orthogonal lines $L_{1 i}=\operatorname{span}\left\{e_{i}\right\}$ where $e_{i}, i=1,2,3$, are unit vectors, and the combined estimator

$$
\frac{1}{3} \sum_{i=1}^{3} \widehat{\Phi}_{3,2,0}\left(X ; L_{1 i}\right)=\frac{\pi}{15} \sum_{i=1}^{3}\left(d\left(x_{+i}, O\right)^{5}+d\left(x_{-i}, O\right)^{5}\right) e_{i}^{2} .
$$

In the special case where $X$ is a ball with radius $R$ centred at the origin

$$
\frac{1}{3} \sum_{i=1}^{3} \widehat{\Phi}_{3,2,0}\left(X ; L_{1 i}\right)=\frac{2 \pi}{15} R^{5} \sum_{i=1}^{3} e_{i}^{2}
$$

This estimator takes the form of $(2 \pi / 15) R^{5}$ times an orthogonal random matrix with known distribution. The mean of this matrix is the identity matrix $I_{3}$. A spectral decomposition of this estimator allows us to exactly reconstruct the ball. For the estimation of functionals of the volume tensor of rank two such as eigenvalues or eigenvectors, it is for general convex bodies preferable to work with more than three lines; cf. Section 6.3 .

The volume tensor of rank two may also be estimated from either isotropic random planes or vertical random planes through $O$. In the case of vertical random planes, we have the following estimator

$$
\widehat{\Phi}_{3,2,0}\left(X ; L_{2(1)}\right)=\frac{\pi}{2} \int_{X \cap L_{2(1)}} x^{2} d\left(x, L_{1}\right) \lambda_{2}(\mathrm{~d} x) .
$$

The estimator based on measurements on one plane is singular. Measurements on at least two systematic vertical random planes should be used.

\subsubsection{Surface Tensors of Rank Two}

The result in Eq. 4 of Proposition 1 contains as special cases estimators of two surface tensors of rank two, $\Phi_{2,2,0}(X)$ and $\Phi_{2,1,1}(X)$. Both estimators use information in an isotropic random plane $L_{2}$ through $O$ and involve integration over all lines in the section plane $L_{2}$, intersecting $X$. At the moment, it is an open question whether these integrals may be expressed in a closed form that is suitable for estimation purposes.

In Auneau-Cognacq et al. (2013, Section 7), it is shown that the remaining surface tensor of rank two, $\Phi_{2,0,2}(X)$, can be expressed as a rotational integral with respect 
to planes through $O$. This result may be used to construct an estimator of $\Phi_{2,0,2}(X)$ of a similar type as the estimators mentioned above for $\Phi_{2,2,0}(X)$ and $\Phi_{2,1,1}(X)$.

\section{A Simulation Study}

The simulation study focuses on the local stereological estimators that are based on measurements on lines, i.e. the estimators in Eqs. 8 and 9 that can be combined to yield an estimator of the centre of gravity and the estimator in Eq. 10 of the volume tensor of rank two.

\subsection{Systematic Sampling on the Sphere}

Taking a systematic random sample of an interval $[a, b]$ on the real line is straight forward. Let $U$ be uniformly distributed in $[0,1]$ and set $x_{i}=a+(U+i)(b-a) / n$, $i=0, \ldots, n-1$, where $n \in \mathbb{N}$. Then $\left\{x_{i}\right\}_{i=0, \ldots, n-1}$ is a systematic random sample of $[a, b]$ with $n$ points. On the unit sphere $\mathbb{S}^{2}$ it is more difficult to specify what we mean by a systematic random sample.

Suppose we are given a set of $n$ points $\left\{x_{i}\right\}_{i=0, \ldots, n-1} \subseteq \mathbb{S}^{2}$ that are equally spaced in some reasonable sense. It is clear that we can create a systematic random sample from these points by rotating them with a uniform random rotation matrix $\mathbf{R} \in$ $S O$ (3). However, there are many ways to sensibly define equally spaced points on the sphere. For our simulation study we have decided to follow the ideas of Leopardi (2006). He defines so-called recursive zonal equal area partitions. They are partitions of $\mathbb{S}^{2}$ into $n$ regions $A_{k}$ of equal area and small diameter. The regions produced by the algorithm are rectilinear in spherical polar coordinates. We take $x_{k}$ to be the point with spherical coordinates equal to the mid range of latitudes and longitudes within $A_{k}$, respectively. As we are interested in systematic random samples of lines through the origin rather than points in $\mathbb{S}^{2}$, we note that it is easy to modify Leopardi's algorithm for even numbers $n=2 N$ such that for each $x_{k} \in\left\{x_{i}\right\}_{i=0, \ldots, 2 N-1}$ we also have $-x_{k} \in\left\{x_{i}\right\}_{i=0, \ldots, 2 N-1}$. We can identify each pair $\left(x_{k},-x_{k}\right)$ with a line through the origin.

When we speak of a systematic set of lines (through the origin) in the sequel, we are referring to the above construction. Figure 1 illustrates systematic random sets of $N=3,7,20$ lines, respectively.
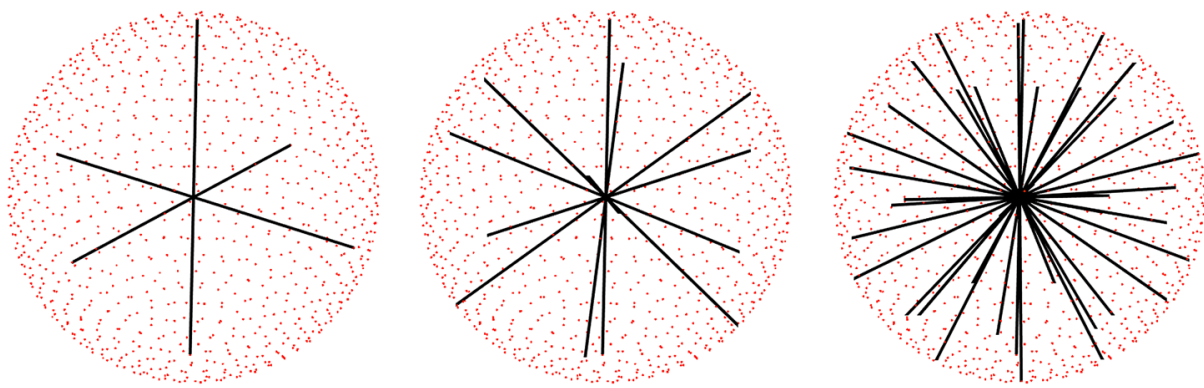

Fig. 1 Systematic sets of $N=3,7,20$ lines, respectively. The red dots visualise the unit sphere $\mathbb{S}^{2}$ 


\subsection{Estimation of the Centre of Gravity}

The two bodies $X$ that we have considered as examples are an ellipsoid with semiaxis lengths $a=7, b=6, c=5$, and a circular cylinder with height $2 h=6$ and radius $r=2$. Both bodies are centred at the origin.

For the estimation of the centre of gravity, we need to choose the origin of our coordinate system. In applications, one will aim to choose this origin as close as possible to the true centre of gravity. Therefore, we model the initial guess by a truncated normally distributed random vector $\mathbf{O}$ with mean zero and covariance matrix $\sigma^{2} I_{3}$.

We chose $\sigma=0.3 c$ for the ellipsoid, and $\sigma=0.3 r$ for the cylinder, and truncated the distribution outside a ball centred at the origin with radius $c$ or $r$ for the ellipsoid and cylinder, respectively. This means that $\mathbf{O} \in X$ always holds. Let $\left\{\mathbf{L}_{1 i}\right\}_{i=0, \ldots, N-1}$ be a systematic random set of $N$ lines through $\mathbf{O}$. Each line $\mathbf{L}_{1 i}$ is spanned by a unit vector $\mathbf{e}_{i}$. As $X$ is convex and $\mathbf{O} \in X$, there are two endpoints $\left(x_{+i}, x_{-i}\right)$ of the line
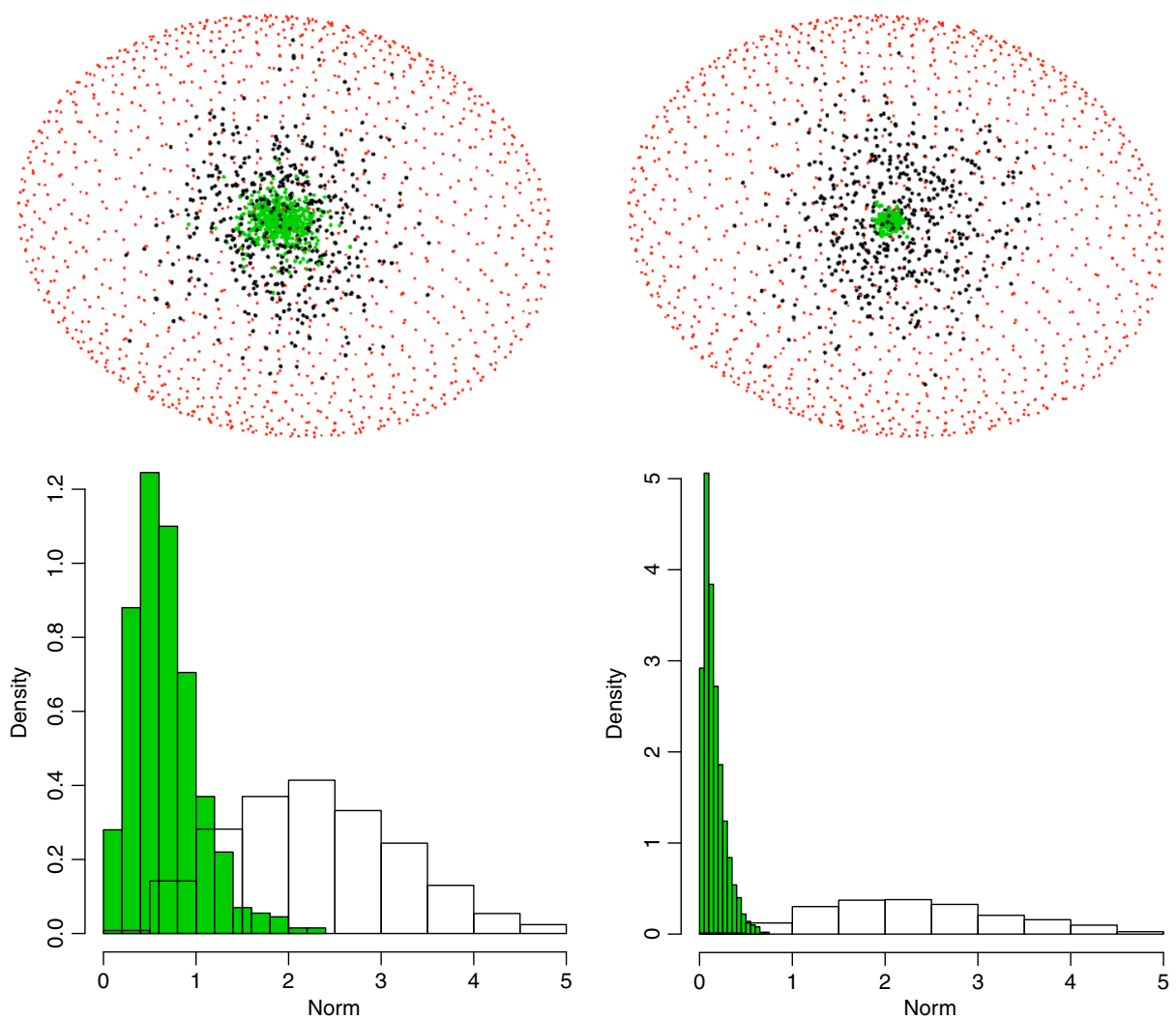

Fig. 2 Estimation of the centre of gravity, using systematic random sets of $N=3$ or $N=7$ lines for the left and right panels, respectively. In the top row, the red dots visualise the ellipsoid; the black dots are the initially chosen origins distributed according to a truncated trivariate normal distribution. The green dots show the estimated centres of gravity. The histograms in the second row show the distribution of the norms of the initially chosen origins (white bars) and of the estimated centres of gravity (green bars) 
segment $X \cap \mathbf{L}_{1 i}$, where $x_{+i}$ points in the same direction as $\mathbf{e}_{i}$. We estimate the centre of gravity $C(X)=\Phi_{3,1,0}(X) / \Phi_{3,0,0}(X)$ by

$$
\widehat{C}(X)=\frac{\frac{\pi}{2} \frac{1}{N} \sum_{i=0}^{N-1}\left(d\left(x_{+i}, \mathbf{O}\right)^{4}-d\left(x_{-i}, \mathbf{O}\right)^{4}\right) \mathbf{e}_{i}}{\frac{2 \pi}{3} \frac{1}{N} \sum_{i=0}^{N-1}\left(d\left(x_{+i}, \mathbf{O}\right)^{3}+d\left(x_{-i}, \mathbf{O}\right)^{3}\right)}
$$

combining Eqs. 8 and 9. The error that is introduced by approximating the integrals by discrete sums depends on the size $N$ of the systematic sample but also on the algorithm chosen to create systematic samples on the sphere; cf. Section 6.1. It is the subject of future research to assess the precision of the proposed estimators.
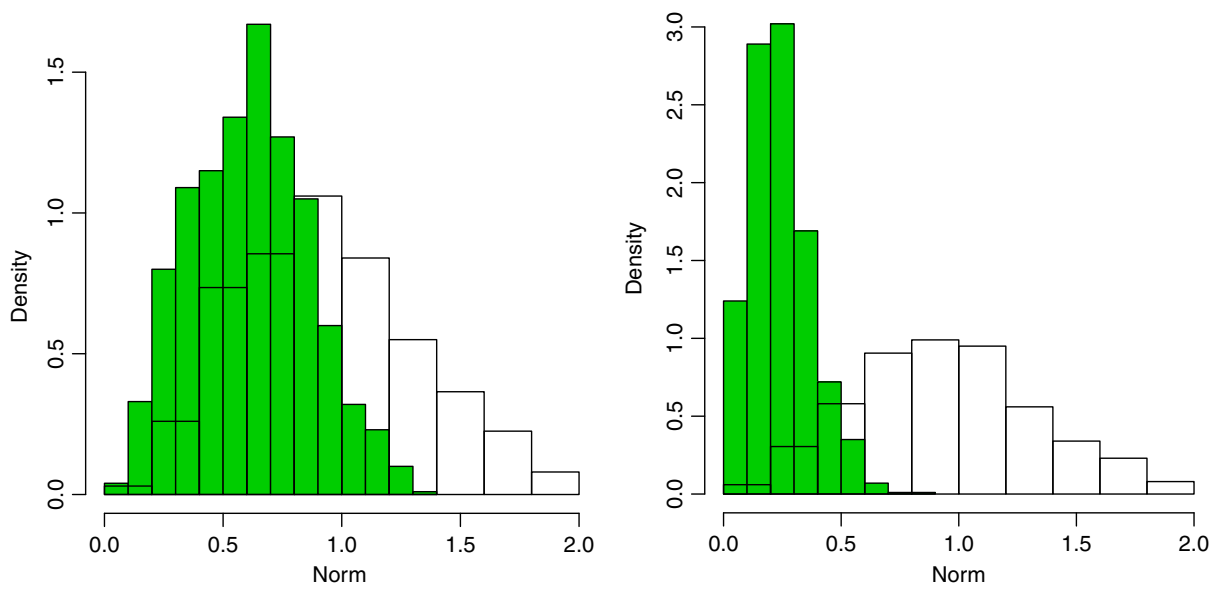

Fig. 3 Estimation of the centre of gravity, using systematic random sets of $N=3$ or $N=7$ lines for the left and right panels, respectively. In the top row, the red dots visualise the cylinder; the black dots display the initially chosen origins distributed according to a truncated trivariate normal distribution. The green dots show the estimated centres of gravity. In the second row, the histograms display the norms of the initial origins (white bars) and the estimated centres of gravity (green bars) 
The results of 500 simulations are visualised in Figs. 2 and 3 for the cylinder and ellipsoid, respectively. For both bodies the simulation results are promising. The estimation procedure generally moves the initially chosen origin towards the true centre of gravity of the body. However, the results for the ellipsoid are better than for the cylinder.

\subsection{Estimation of the Volume Tensor of Rank Two}

The volume tensor of rank two of a body $X$ is defined relative to the origin of the coordinate system. If we want to use volume tensors to characterise the shape and
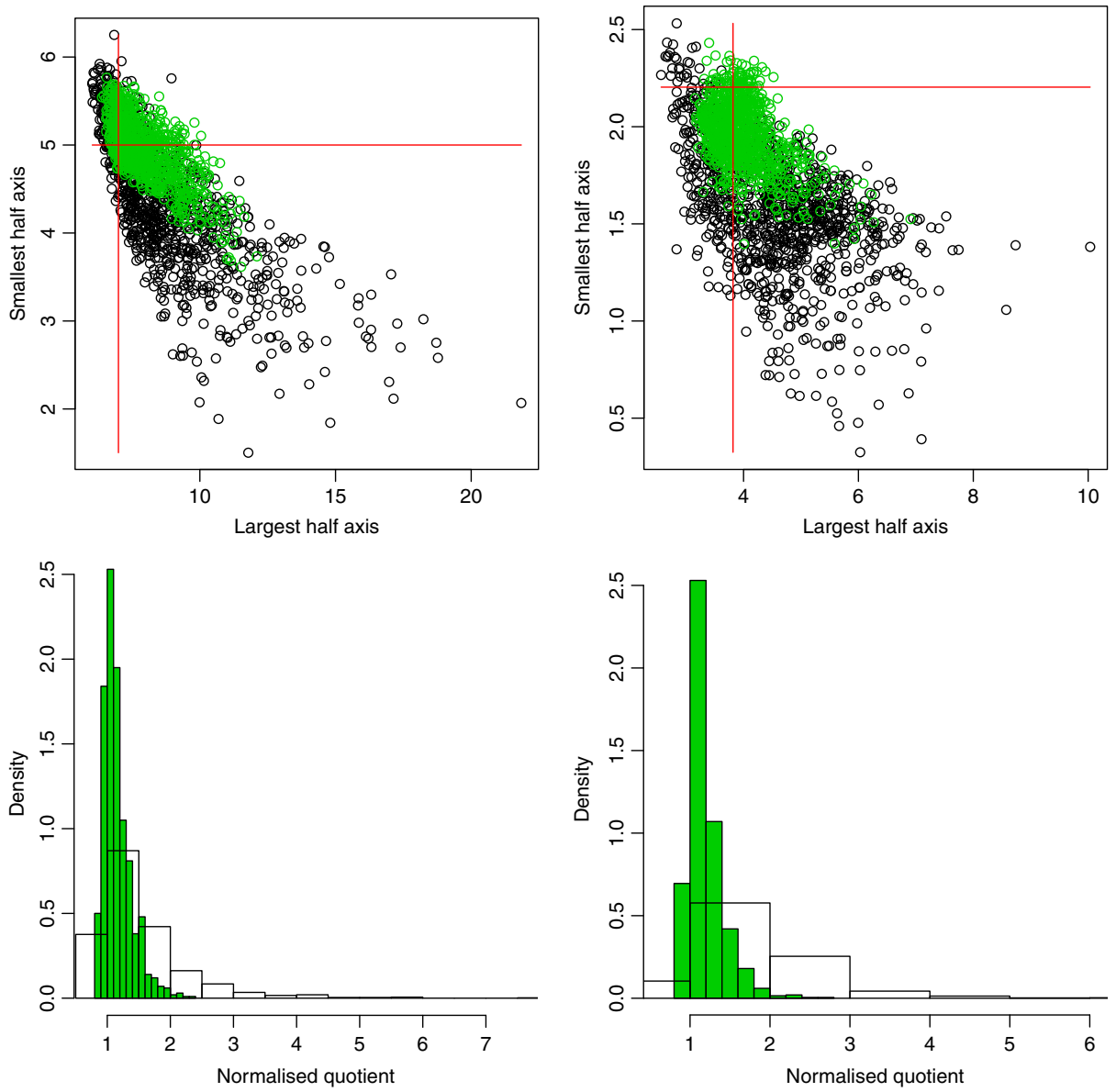

Fig. 4 Smallest and largest semiaxes of the estimated associated ellipsoid. The panels in the top row show scatter plots of the largest and smallest semiaxes for $N=3$ lines (in black) and for $N=7$ lines (in green). The red lines mark the true values. In the bottom row, histograms of the quotient of the largest over the smallest estimated semiaxes normalised by the true quotient (i.e. the target value is 1$)$ are displayed as empty bars $(N=3)$ or green bars $(N=7)$. The left column corresponds to the example where $X$ is an ellipsoid, whereas the right column corresponds to the cylinder. The histogram for the cylinder for $N=3$ was cut off at 6 . There were observed normalised quotients as large as 10.73 
orientation of particles in a particle population, we need to specify a centre point (or origin) for each particle in a unique way. We chose to consider the centre of gravity as the "true" centre of the bodies (or particles) under consideration.

The volume tensor of rank two is a symmetric, positive definite $3 \times 3$ matrix $M$. For each such matrix $M$ it is possible to construct an ellipsoid $Y$ such that $\Phi_{3,2,0}(Y)=$ $M$; cf. Section 3. We call this ellipsoid $Y$ the associated ellipsoid to $M$ or to $X$, if $\Phi_{3,2,0}(X)=M$. This association may be regarded as an ellipsoidal approximation to the original body $X$. If $X$ is an ellipsoid itself then it is equal to its associated ellipsoid. When characterizing the shape of $X$ through the volume tensor one can consider the largest and smallest eigenvalue of $\Phi_{3,2,0}(X)$ or their quotient. Alternatively, one can also compare the smallest and largest semiaxes of the associated ellipsoid to
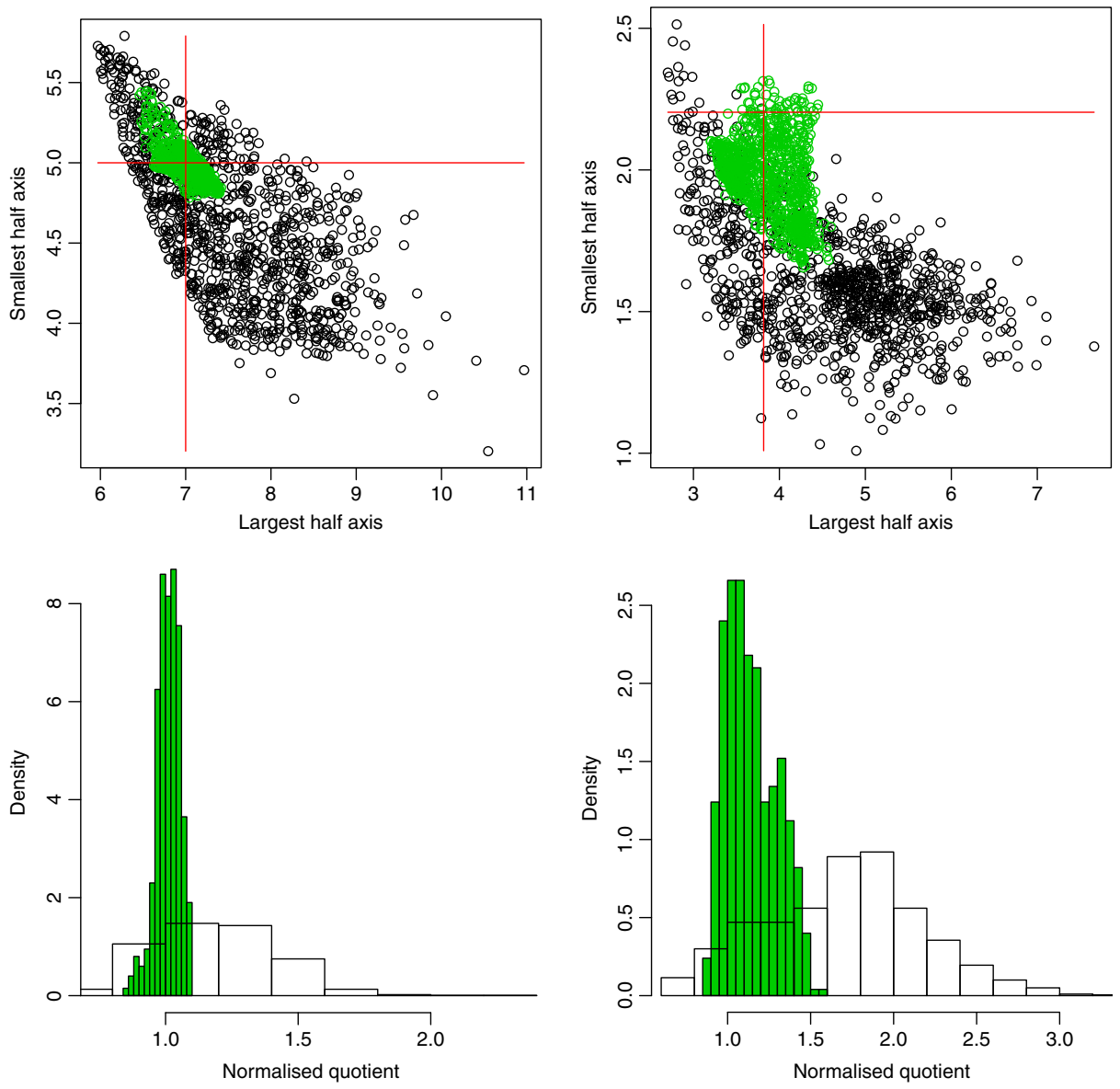

Fig. 5 Smallest and largest semiaxes of the estimated associated ellipsoid for the two-step procedure. The scatter plots in the top row show the largest vs. smallest semiaxes for $N=3$ lines (in black) and for $N=7$ lines (in green). The red lines mark the true values. Histograms of the normalised quotient of the largest over the smallest estimated semiaxes are displayed in the bottom row. Empty bars correspond to $N=3$, whereas green bars correspond to $N=7$. The normalization yields a target value of 1 . The left column corresponds to the ellipsoid, whereas the right column corresponds to the cylinder 
$\Phi_{3,2,0}(X)$, which is our method of choice. The reason for this choice is primarily that the lengths of the semiaxes of the associated (or approximating) ellipsoid are easier to interpret geometrically. The orientation of $X$ is characterised by the eigenvectors of $\Phi_{3,2,0}(X)$ which are the same as the directions of the semiaxes of the associated ellipsoid.

The tensor $\Phi_{3,2,0}(X)$ can be estimated from isotropic lines through the origin using Eq. 10. We assumed that the origin is initially chosen as $\mathbf{O}$, which has a trivariate truncated normal distribution as specified in Section 6.2. In the first part of the simulation study, we estimated $\Phi_{3,2,0}(X)$ by

$$
\widehat{\Phi}_{3,2,0}(X)=\frac{1}{N} \frac{\pi}{5} \sum_{i=1}^{N}\left(d\left(x_{+i}, \mathbf{O}\right)^{5}+d\left(x_{-i}, \mathbf{O}\right)^{5}\right) \mathbf{e}_{i}^{2}
$$

from a systematic random set of lines $\left\{\mathbf{L}_{1 i}\right\}_{i=0, \ldots, N-1}$ of size $N=3$ or $N=7$ with respect to $\mathbf{O}$ using Eq. 10 and the notation introduced in Section 6.2. We used the ellipsoid and the cylinder described in Section 6.2 as examples for $X$. The results of 1000 simulations are given in terms of the associated ellipsoid in Fig. 4. The
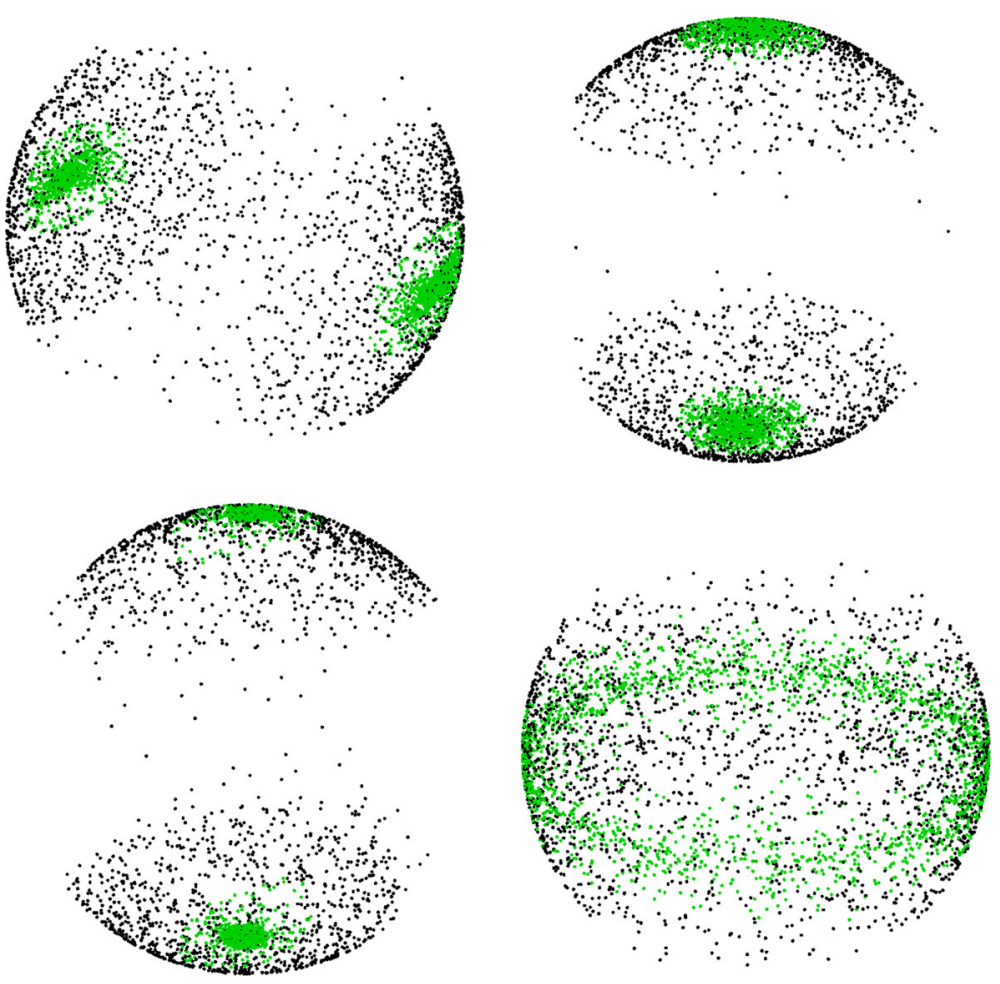

Fig. 6 Direction of the estimated largest and smallest semiaxes for the two-step procedure as points on the unit sphere. The black points correspond to $N=3$ lines, whereas the green points correspond to $N=7$ lines. In the left and right column the results for the ellipsoid and cylinder are displayed, respectively. The top row corresponds to the direction of the largest semiaxis, whereas the bottom row corresponds to the direction of the smallest semiaxis 
eigenvalues of the associated ellipsoid were calculated from $\widehat{\Phi}_{3,2,0}(X)$ using the formulas in Section 3.

In the second part of the simulation study, we used a two-step procedure. First, the centre of gravity was estimated by $\widehat{C}(X)$ starting from $\mathbf{O}$ as described in Section 6.2 using a systematic random set of $N$ lines. Then an independent systematic random set of lines of the same size, centred at $\widehat{C}(X)$, was used to estimate $\widehat{\Phi}_{3,2,0}(X)$ by Eq. 11 with $\mathbf{O}$ replaced by $\widehat{C}(X)$. The results of 1000 simulations are displayed in Figs. 5 and 6.

In summary, the two-step procedure clearly outperforms the first approach. It works well for detecting anisotropy for both the cylinder and the ellipsoid. In Fig. 5 it can be seen that neither body is ever classified as spherical in 1000 simulations. There is a marked improvement in estimating the degree of anisotropy by the quotient of the half axes when working with $N=7$ instead of $N=3$ lines. This increase in precision is also pronounced for the estimation of the direction of the extremal semiaxes. The values of the smallest and largest semiaxes are captured well by the estimates, for both, the ellipsoid and the cylinder.

\section{Discussion}

Minkowski tensors are a natural extension of intrinsic volumes. They provide descriptors of size, location, shape and orientation of convex bodies. In material science their usefulness as summary statistics of populations of shapes has been demonstrated in the past. In these applications it is usually possible to observe the entire structure and detect it automatically. In many stereological applications, especially in the biosciences, it is often impossible to have access to the entire boundary of a particle of interest.

We believe that Minkowski tensors can provide powerful descriptors of size, location, shape and orientation in the biosciences. Therefore, this paper presents local stereological methods to estimate the tensors based on measurements on sections or lines through the particle. Some of the presented estimators have a long history and have been widely applied for decades, whereas others are new. We have chosen to present both, old and new estimators, as the tensorial perspective provides a unified framework for seemingly different approaches.

In this paper, we have restricted our presentation to convex bodies. However, some of the old estimators presented here have been considered for more general set classes. For example, the nucleator (Section 5.1.1) can be defined for compact sets $X \subseteq \mathbb{R}^{3}$. The simple formula in Eq. 8 continues to hold as long as $X$ is starshaped with respect to the origin. If $X$ is an element of the convex ring, i.e. a finite union of convex bodies, then $X \cap L_{1}$ will be a finite union of line segments and Eq. 8 has to be replaced with an alternating sum of the distances from the origin to the endpoints of the line segments. The isotropic rotator (Section 5.1.2) and the vertical rotator (Section 5.1.3) have also been considered for general compact sets. CruzOrive (2005) has considered the flower estimator (Section 5.1.4) for compact sets with piecewise smooth boundary. A comprehensive overview of possible extensions of the flower estimator to more general classes of sets is given in Thórisdóttir and Kiderlen (2013). 
It is possible to define generalised curvature measures for sets $X$ of positive reach; see for example Zähle (1987) for details. Therefore, Minkowski tensors can be defined for such set classes, and it would be interesting to develop local stereological estimators in this setting. However, the results are likely to become much more technical, and the interpretation of the tensors for biological applications will pose a challenge in itself.

In a simulation study we investigate the performance of two of the newly proposed estimators on two example shapes. The results are promising, however, many applied statistical questions remain to be addressed. For example, the volume tensor of the particles is supposed to be used to discriminate whether a particle population shows a preferred orientation or not. For this purpose, one needs to develop statistical tests. In this context, it may be preferable to address the estimators in a model based framework in order to have a suitable model under the null hypothesis. We believe, that these questions should be answered in close collaboration with practitioners as in different applications, different functionals of the volume tensor of rank two may have a meaningful interpretation.

Acknowledgements This work has been supported by Centre for Stochastic Geometry and Advanced Bioimaging, funded by a grant from The Villum Foundation.

\section{References}

Auneau-Cognacq J, Ziegel J, Jensen EBV (2013) Rotational integral geometry of tensor valuations. Adv Appl Math 50:429-444

Beisbart C, Dahlke R, Mecke KR, Wagner H (2002) Vector- and tensor-valued descriptors for spatial patterns. In: Morphology of condensed matter, vol 600. Lecture notes in physics. Springer, Berlin, pp 249-271

Beisbart C, Barbosa MS, Wagner H, da F Costa L (2006) Extended morphometric analysis of neuronal cells with Minkowski valuations. Eur Phys J B 52:531-546

Beneš V, Jiruše M, Slámová M (1997) Unfolding the trivariate size-shape-orientation distribution of spheroidal particles with application. Acta Mater 45(3):1105-1113

Cruz-Orive LM (1976) Particle size-shape distributions: the general spheroid problem. i. Mathematical model. J Microsc 107:235-253

Cruz-Orive LM (1978) Particle size-shape distributions: the general spheroid problem. II. Stochastic model and practical guide. J Microsc 112:153-167

Cruz-Orive LM (2005) A new stereological principle for test lines in three-dimensional space. J Microsc 219:18-28

Cruz-Orive LM (2008) Comparative precision of the pivotal estimators of particle size. Image Anal Stereol 27:17-22

Cruz-Orive LM (2011) Flowers and wedges for the stereology of particles. J Microsc 243:86-102

Cruz-Orive LM (2012) Uniqueness properties of the invariator, leading to simple computations. Image Anal Stereol 31:89-98

Cruz-Orive LM, Hoppeler H, Mathieu O, Weibel ER (1985) Stereological analysis of anisotropic structures using directional statistics. J Roy Statist Soc Ser C 34:14-32

Denis EB, Barat C, Jeulin D, Ducottet C (2008) 3D complex shape characterizations by statistical analysis: Application to aluminium alloys. Mater Charact 59:338-343

Dvořák J, Jensen EBV (2012) On semi-automatic estimation of surface area. CSGB Research Report 12-06, Centre for Stochastic Geometry and Advanced Bioimaging, Department of Mathematics, Aarhus University, Denmark. Submitted

Gokhale M (1996) Estimation of bivariate size and orientation distribution of microcracks. Acta Mater 44(2):475-485

Gundersen HJ (1988) The nucleator. J Microsc 151:3-21

Hansen LV, Nyengaard JR, Andersen JB, Jensen EBV (2011) The semi-automatic nucleator. J Microsc 242:206-215 
Hug D, Schneider R, Schuster R (2008) Integral geometry of tensor valuations. Adv Appl Math 41:482-509

Jensen EB, Gundersen HJG (1987) Stereological estimation of surface area of arbitrary particles. Acta Stereol 6:25-30

Jensen EBV (1998) Local stereology. World Scientific, London

Jensen EBV, Gundersen HJ (1993) The rotator. J Microsc 170:35-44

Leopardi P (2006) A partition of the unit sphere into regions of equal area and small diameter. Electron Trans Numer Anal 25:309-327 (electronic)

Pawlas Z, Nyengaard JR, Jensen EBV (2009) Particle sizes from sectional data. Biometrics 65:216224

Schröder-Turk GE, Kapfer SC, Breidenbach B, Beisbart C, Mecke K (2011a) Tensorial Minkowski functionals and anisotropy measures for planar patterns. J Microsc 238:57-74

Schröder-Turk GE, Mickel W, Kapfer SC, Klatt MA, Schaller FM, Hoffmann MJF, Kleppmann N, Armstrong P, Inayat A, Hug D, Reichelsdorfer M, Peukert W, Schwieger W, Mecke K (2011b) Minkowski tensor shape analysis of cellular, granular and porous structures. Adv Mater 23:25352553

Thórisdóttir O, Kiderlen M (2013) The invariator principle in convex geometry (in preparation)

Zähle M (1987) Curvatures and currents for unions of sets with positive reach. Geom Dedicata 23:155-171 